\title{
Differentiation of human amniotic epithelial cells into osteoblasts is induced by mechanical stretch via the Wnt/ $\boldsymbol{\beta}$-catenin signalling pathway
}

\author{
FUJUN LUAN $^{1 *}$, KUNLONG MA $^{1 *}$, JIA MAO $^{2}$, FAN YANG $^{1}$, MINGHUA ZHANG $^{1}$ and HEXU LUAN ${ }^{1}$ \\ Departments of ${ }^{1}$ Orthopedics and ${ }^{2}$ Dermatology, Yongchuan Hospital of Chongqing Medical University, \\ Chongqing 402160, P.R. China
}

Received November 12, 2016; Accepted March 2, 2018

DOI: $10.3892 / \mathrm{mmr} .2018 .9571$

\begin{abstract}
Human amniotic epithelial cells (hAECs) have recently been recognized as a potential source of stem cells. The present study was designed to investigate the effects of mechanical stretch on the osteogenic differentiation of hAECs. As it has been previously reported that the physical environment is an important factor in maintaining the phenotype and functionality of differentiated cells, mechanical stretch was use to mimic the mechanical environment in the present study, with the following parameters: $5 \%$ elongation of the hAECs at a frequency of $0.5 \mathrm{~Hz}$, with evaluation at 2, 6, 12 and $24 \mathrm{~h}$ time points. The osteogenic differentiation process of the hAECs followed by mechanical stimulation was evaluated by reverse transcription-quantitative polymerase chain reaction (RT-qPCR), western blotting and immunocytochemistry. Additionally, in a parallel study, a runt-related transcription factor 2 (Runx2)/core binding factor $\alpha 1$ (Cbfa1)-specific short hairpin RNA (shRNA) plasmid vector and a scrambled shRNA control plasmid was constructed for transfection into the hAECs prior to mechanical stimulation. The cultured hAECs exhibited a cobblestone-shaped epithelial-like phenotype and were positive for stage-specific embryonic antigen-4, cytokeratin-19, cluster of differentiation 44 and octamer-binding protein 4 , as detected by flow cytometry, western blotting or confocal microscopy. The qPCR and western blotting data demonstrated that the mRNA and protein expression levels of Runx2/Cbfa1, alkaline phosphatase and osteocalcin were upregulated compared with the control group following stretching and they peaked at $12 \mathrm{~h}$. These results
\end{abstract}

Correspondence to: Dr Hexu Luan, Department of Orthopedics, Yongchuan Hospital of Chongqing Medical University, 439 Xuanhua Road, Yongchuan, Chongqing 402160, P.R. China

E-mail: xiaoyuer20131213@126.com

*Contributed equally

Key words: human amniotic epithelial cells, stem cell, mechanical stretch, osteogenic differentiation, cell therapy indicated that the osteogenic differentiation of the hAECs was induced by mechanical stimuli. Additionally, the mRNA and protein expression levels of $\beta$-catenin and cyclin $\mathrm{D}$ were increased significantly following stretching; however, they were decreased following Runx2/Cbfa1-shRNA transfection as observed by RT-qPCR and western blotting. These results suggested that the $W n t / \beta$-catenin pathway may have an important role in mechanical stretch-induced osteogenic differentiation of the hAECs. Furthermore, the combination of stretch and osteogenic induction medium had synergistic effects on the osteogenic differentiation. The results of the present study demonstrated that mechanical stimuli have an important role in osteogenic differentiation of hAECs via the Wnt/ $\beta$-catenin signalling pathway, which may be a potential therapeutic strategy in bone regenerative medicine.

\section{Introduction}

Mechanical stimulation is an important environmental factor that influences bone metabolism (1). The molecular signals driving the regenerative process in distraction osteogenesis involves a complex system of cellular behaviours triggered by mechanical strain (1). Previously, attention has been focused on the role of mechanical forces in the differentiation of stem cells, including the differentiation of mesenchymal stem cells (MSCs) into osteoblasts (2,3). However, whether mechanical stimuli can modulate the osteogenic differentiation of human amniotic epithelial cells (hAECs) remains unknown.

hAECs can be derived from the full-term placenta and express embryonic stem cell (ESC) markers, including stage-specific embryonic antigen-4 (SSEA-4) and octamer-binding protein 4 (Oct-4) $(4,5)$. According to Miki et al (5), the amnion is derived from the totipotent epiblast and is considered an alternative source of stem cells due to the expression of SSEA-4. Furthermore, as Toda et al (6) reported that various advantages make the amnion a potentially useful and noncontroversial source of cells for transplantation and regenerative medicine. The considerable advantages described in detail were as follows: hAECs can differentiate into all three germ layers; they demonstrate low immunogenicity and anti-inflammatory functions; and they prompt no ethical concerns due to not requiring the sacrifice of human embryos 
for their isolation, therefore avoiding the current controversies associated with the use of human ESCs $(5,6)$.

hAECs have the potential to differentiate into all three germ layers: Endoderm (liver, pancreas), mesoderm (cardiomyocyte) and ectoderm (neural cells) in vitro $(7,8)$. According to Kakishita et al (9), AECs can differentiate into mature neural cells that synthesize and release acetylcholine, norepinephrine and dopamine. Wlodarski et al (10) demonstrated that WISH cells (an amniotic epithelial cell line) grafted intramuscularly into rats or into Syrian hamsters survived 14 days and induced both cartilage and bone formation. It has also been reported that hAECs can differentiate into osteoblasts following stimulation with a pulsed electromagnetic field (11). Therefore, stimulation of hAECs in this manner has been proposed as a potential source of stem cells for cell therapy.

Previously, stem cell-based therapies have been utilized for their ability to regenerate osteoblasts and have been demonstrated to be efficacious in experimental contexts and human disease. In addition, dexamethasone, $\beta$-glycerol phosphate and ascorbic acid have been identified as osteogenic differentiation inducers in hAECs (12). Furthermore, reports of hAECs differentiating into osteoblasts upon induction by mechanical stretching are rare. In the present study, the hypothesis that mechanical stretching serves important roles in the process of osteogenic differentiation of hAECs was confirmed.

\section{Materials and methods}

Isolation and culture of hAECs. Placentas were donations from six patients (from February 15th 2015 to August 4th 2017; mean age, 32 \pm 4.5 ) of Yongchuan Hospital of Chongqing Medical University (Chongqing, China). All patients gave written informed consent. The present study was performed according to the Declaration of Helsinki and approved by the Medical Ethics Committee of the Yongchuan Hospital of Chongqing Medical University.

As described by Miki et al (13), the amnion layer was mechanically peeled from human placenta and digested with $0.05 \%$ trypsin $+0.02 \%$ EDTA for $30 \mathrm{~min}$ at $37^{\circ} \mathrm{C}(\mathrm{Gibco}$; Thermo Fisher Scientific, Inc., Waltham, MA, USA). Following filtration with 200-mesh filter and centrifugation at $200 \mathrm{x} g$ for 5 min at $4^{\circ} \mathrm{C}$, the cells were cultured in low glucose Dulbecco's modified Eagle's medium (DMEM; Gibco; Thermo Fisher Scientific, Inc.) at a density of $1 \times 10^{5}$ cells $/ \mathrm{cm}^{2}$ and placed in a $37^{\circ} \mathrm{C}$ and $5 \% \mathrm{CO}_{2}$ humidified incubation chamber. The medium was supplemented with $5 \%$ foetal bovine serum (FBS; Gibco; Thermo Fisher Scientific, Inc.), $2 \mathrm{mmol} /$ l-glutamine, $1 \%$ non-essential amino acids and $55 \mu \mathrm{mol} / \mathrm{l}$ $\beta$-mercaptoethanol. New culture medium was supplied after $24 \mathrm{~h}$ of subculture. Experiments were performed once the cells were fused into a single layer and reached $80-90 \%$ confluence.

Identification of hAECs. The hAECs were identified immunocytochemically by the specific epithelial cell marker cytokeratin-19 (CK19) and the ESC marker Oct-4, the cell surface marker cluster of differentiation 44 (CD44), and the stem cell surface marker SSEA-4 (all Abcam, Cambridge, $\mathrm{UK})$.

hAECs (3rd passage) were seeded onto cover slips in 6-well plates. At 80-90\% confluence, they were incubated with primary antibodies (1:300) at $4{ }^{\circ} \mathrm{C}$ and secondary antibodies (Goat anti-Mouse/rabbit IgG (H+L) secondary antibody; Alex Fluor 488/594; 1:200; Jackson) at $25^{\circ} \mathrm{C}$ following a standard immunocytochemistry protocol including fixing with $4 \%$ polyoxymethylene at $4^{\circ} \mathrm{C}$ for $30 \mathrm{~min}$, permeabilizing with $1 \%$ Triton-X100 for $10 \mathrm{~min}$ at $25^{\circ} \mathrm{C}$ and blocking with $5 \%$ bovine serum albumin (BSA; Sigma-Aldrich, Darmstadt, Germany, USA) for $30 \mathrm{~min}$ at $25^{\circ} \mathrm{C}$. Eventually, 4',6-diamidino-2phenylindole (DAPI; 1:1,000; Invitrogen; Thermo Fisher Scientific, Inc.) was used to mark the nucleus for $10 \mathrm{~min}$ at $25^{\circ} \mathrm{C}$. The stained cells were imaged on a confocal inverted microscope (Olympus FluoView FV 1,000; Olympus Corporation, Tokyo, Japan).

Mechanical stretch stimulation. In the mechanical stretch group, the 3rd to 5th passage hAECs were seeded reached $60-70 \%$ confluency on silicone membranes coated with collagen type I, transferred onto a uniaxial stretch chamber (size, $4.0 \times 7.0 \mathrm{~cm}$ ), the maximum uniaxial stretched length is $7.35 \mathrm{~cm}$, using a machine similar to that in Hata et al (14) and stretched for 2, 6, 12 and $24 \mathrm{~h}$ with 5\% elongation at a frequency of $0.5 \mathrm{~Hz}(15,16)$. The positive control group was treated with osteogenic induction medium (OM; DMEM supplemented with $100 \mathrm{nmol} / 1$ dexamethasone, $50 \mathrm{mg} / \mathrm{l}$ ascorbic acid and $5 \mathrm{mmol} / 1 \beta$-glycerophosphate) for 21 days. The stretch-biochemical combined stimulation group was treated with stretching with $5 \%$ elongation at $0.5 \mathrm{~Hz}$ for 2, 6, 12 and $24 \mathrm{~h}$ following 21 days of OM stimulation. The cells were collected using TRIzol ${ }^{\mathrm{TM}}$ (Thermo Fisher Scientific, Inc.) or radioimmunoprecipitation assay (RIPA) lysis buffer (Beyotime Institute of Biotechnology, Haimen, China) at each time point. Each experiment was conducted in five replicates.

RNA interference. $4 \mu \mathrm{g}$ of runt-related transcription factor 2 (Runx2)/core binding factor $\alpha 1$ (Cbfa1)-specific short hairpin RNA (shRNA) plasmid and $4 \mu \mathrm{g}$ of scrambled shRNA control plasmid was constructed, followed by cationic lipid (Lipofectamine $^{\mathrm{TM}} 2000$ Invitrogen; Thermo Fisher Scientific, Inc.) transfection for $48 \mathrm{~h}$ at $37^{\circ} \mathrm{C}$. OM induction and stretch was performed following transfection for $24 \mathrm{~h}$. The sequences of Runx2/Cbfa1-shRNA and scrambled shRNA, designed based on NCBI GeneBank data, are demonstrated in Table I.

Reverse transcription-quantitative polymerase chain reaction $(R T-q P C R)$. The hAECs in each group were collected with TRIzol and the corresponding RNA samples were obtained following extraction and purification. For extraction, $1 \mathrm{ml}$ TRIzol was used to collect total RNA and $0.2 \mathrm{ml}$ chloroform was added to each sample to extract RNA. Following incubation at $4^{\circ} \mathrm{C}$ for $5 \mathrm{~min}$, samples were centrifuged at $12,000 \mathrm{x} \mathrm{g}$ for $15 \mathrm{~min}$ at $4^{\circ} \mathrm{C}$. Then, the upper aqueous fraction was collected by the addition of $0.5 \mathrm{ml}$ ethanol. Followed centrifugation at $12,000 \mathrm{x} \mathrm{g}$ for $15 \mathrm{~min}$ at $4^{\circ} \mathrm{C}, 75 \%$ ethanol was used to purify the extracted RNA. Following air-drying, RNA was eluted in $20 \mu \mathrm{l}$ RNase-free water. RNA concentrations were quantified by spectrophotometry (Bio-Rad Laboratories, Inc., Hercules, CA, USA) and cDNA was synthesized using a RT kit (FSQ-101; Toyobo Life Science, Osaka, Japan) at $37^{\circ} \mathrm{C}$ for $5 \mathrm{~min}$ and then $95^{\circ} \mathrm{C}$ for $5 \mathrm{~min}$. To detect the accumulation of PCR products, EvaGreen (Bio-Rad Laboratories, Inc.) with 
Table I. Sequences of Runx2-shRNA and scrambled control.

\begin{tabular}{ll}
\hline Name & \multicolumn{1}{c}{ Sequence } \\
\hline Runx2-shRNA & F: 5'-GATCCCCAGACTTACTGTATGTATAGTTCAAGAGACTATACATACAGTAAGTCTTTTTT \\
GGAAA-3' & R: 5'-AGCTTTTCCAAAAAAGACTTACTGTATGTATAGTCTCTTGAACTATACATACAGTAAG \\
& TCTGGG-3' \\
& F: 5'-GATCCCCTTCTCCGAACGTGTCACGTTTCAAGAGAACGTGACACGTTCGGAGAATTT \\
Control shRNA & TTGGAAA-3' \\
& R: 5'-AGCTTTTCCAAAAATTCTCCGAACGTGTCACGTTCTCTTGAAACGTGACACGTTCGG \\
& AGAGG-3' \\
\hline
\end{tabular}

Runx2, Runt-related transcription factor 2; shRNA, short hairpin RNA.

Table II. Primer sequences for reverse transcription-quantitative polymerase chain reaction.

\begin{tabular}{lcc}
\hline Gene & GenBank ID & Primer sequence (5'-3') \\
\hline ALP & NM_000478 & F: 5'GGAACTCCTGACCCTTGACC 3' \\
& & R: 5'TCCTGTTCAGCTCGTACTGC 3' \\
OC & NM_001199662 & F: 5'AGGGCAGCGAGGTAGTGAA 3' \\
& & R: 5'TCCTGAAAGCCGATGTGGT 3' \\
Runx2/Cbfa1 & NM_001015051 & F: 5'TTACTTACACCCCGCCAGTC 3' \\
$\beta$-catenin & & R: 5'TATGGAGTGCTGCTGGTCTG 3' \\
& NM_020248 & F: 5'CCTATGCAGGGGTGGTCAAC 3' \\
Cyclin D1 & & R: 5'CGACCTGGAAAACGCCATCA 3' \\
$\beta$-actin & NM_053056.2 & F: 5'TTTGTTGTGTGTGCAGGGAG 3' \\
& & R: 5'TTTCTTCTTGACTGGCACGC 3' \\
& NM_001099771 & F: 5'ACTATCGGCAATGAGCGGTTC 3' \\
& & R: 5'ATGCCACAGGATTCCATACCC 3'
\end{tabular}

OC, osteocalcin; ALP, alkaline phosphatase; Runx2, runt-related transcription factor 2; Cbfa1, core binding factor $\alpha 1$.

the CFX-96 ${ }^{\mathrm{TM}}$ (Bio-Rad Laboratories, Inc.) Real-Time PCR Detection System was used followed by 40 cycles of: $95^{\circ} \mathrm{C}$ for $5 \mathrm{~min}, 95^{\circ} \mathrm{C}$ for $10 \mathrm{sec}, 59^{\circ} \mathrm{C}$ for $1 \mathrm{~min}$. The sequences of qPCR primers and program are presented in Table II. The $\beta$-actin gene was used as an internal reference (17).

Western blot analysis. The proteins from each group of hAECs were separately collected in RIPA lysis buffer (Santa Cruz Biotechnology, Inc., Dallas, TX, USA). Following centrifugation at $4^{\circ} \mathrm{C}$ and $16,000 \times \mathrm{g}$ for $10 \mathrm{~min}$, the supernatant proteins were collected. The concentration was determined by the bicinchoninic acid method, followed by loading $20 \mu \mathrm{g}$ total proteins onto the SDS-PAGE gels (4\% separation gel, $5 \%$ spacer gel) and transferred to $0.45 \mu \mathrm{m}$ Immobilon polyvinylidene difluoride membranes (EMD Millipore, Billerica, MA, USA). The membranes were blocked with 5\% BSA in Tris Buffered Saline with Tween ${ }^{\circledR} 20$ (TBST) at $25^{\circ} \mathrm{C}$ for $1 \mathrm{~h}$, incubated with the indicated primary antibodies [anti- $\beta$-catenin $(1: 1,000$; cat. no. ab16051), anti-Runx2 (1:1,000; ab23981), anti-Cyclin D1 (1:1,000; cat. no. ab226977), anti-alkaline phosphatase (1:1,000; cat. no. ab229126) and anti-osteocalcin (1:1,000; cat. no. ab93876; Abcam)] at $4^{\circ} \mathrm{C}$ overnight and then incubated with the corresponding secondary antibodies (Goat anti-mouse/rat secondary antibodies; 1:3,000; cat. no.ZB2305/ZB2306; Zsbio; OriGene Technologies, Inc., Beijing, China) at $25^{\circ} \mathrm{C}$ for $1 \mathrm{~h}$. Images were obtained with an enhanced chemiluminescence kit (Thermo Fisher Scientific, Inc.) on film and the images were captured, and analysed with a gel imaging system (2.0 version of quantity one 1-D, Bio-Rad Laboratories, Inc.).

Statistical analysis. The data are presented as the mean \pm standard deviation. Single-tailed Student's t-test of unpaired data or one-way analysis of variance, followed by the least significance difference performed in SPSS 13.0 software (SPSS, Inc., Chicago, IL, USA) was used for comparisons between groups, $\mathrm{n}=3$. $\mathrm{P}<0.05$ was considered to indicate a statistically significant difference.

\section{Results}

Morphology and characteristics of isolated hAECs. hAECs were successfully isolated from full-term amnion without 

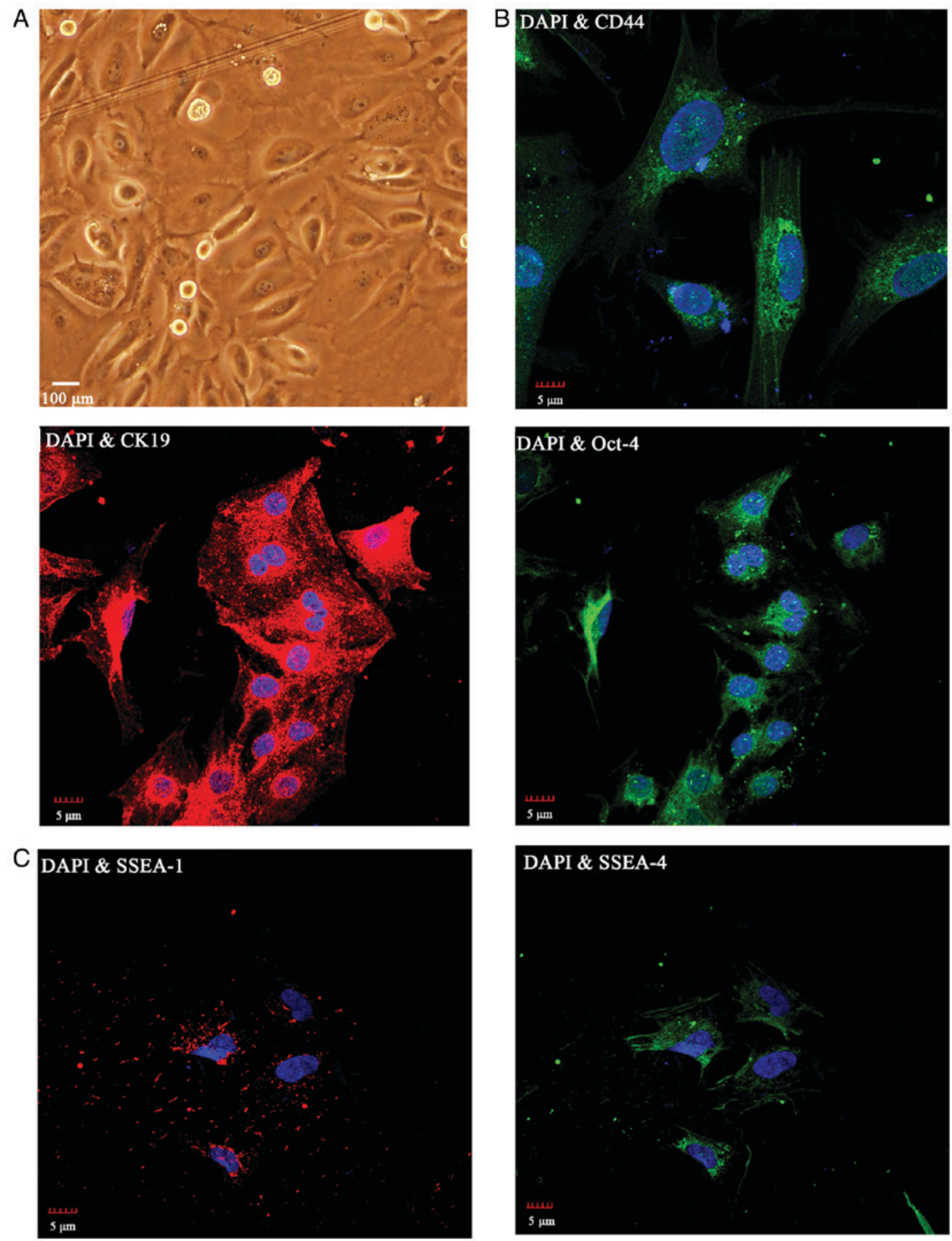

Figure 1. Morphology and characteristics of isolated hAECs. (A) Phase contrast microscopy image of isolated hAECs (x200). (B) Protein expression levels of CK-19, Oct-4 and CD44 as detected by immunocytochemistry. (C) Confocal microscopy (magnification, x600) demonstrated positive expression of the stem cell surface marker SSEA-4 but was negative for SSEA-1. hAECs, human amniotic epithelial cells; CK, cytokeratin; Oct-4, octamer binding protein-4; SSEA, stage-specific embryonic antigen.

disease. Following 3 days in culture, the hAECs demonstrated the typical morphology of cobblestone-shape epithelial-like cells (Fig. 1A).

Expression of the epithelial cell marker CK19, and the ESC markers CD44 and Oct-4 was detected in the primary cultures of hAECs by immunocytochemistry (Fig. 1B). The stem cell surface marker SSEA-4 was highly expressed in the hAECs, as indicated by the confocal images; whereas, SSEA-1 was negative (Fig. 1C). Together, the results suggested that the hAECs that were obtained were CK19-positive and expressed stem cell markers, CD44, Oct-4 and SSEA-4, which is consistent with previous reports $(4,5)$. Based on the DAPI and marker staining that were performed in Fig. 1, the majority of the cells that were isolated were indeed hAECs.

Stretch regulates the osteogenic differentiation of AECs. To determine whether the mechanical stimulation affected the osteogenic differentiation of the hAECs, the protein level of osteocalcin was detected by immunocytochemistry. The data demonstrated that the osteocalcin (OC) level was induced 


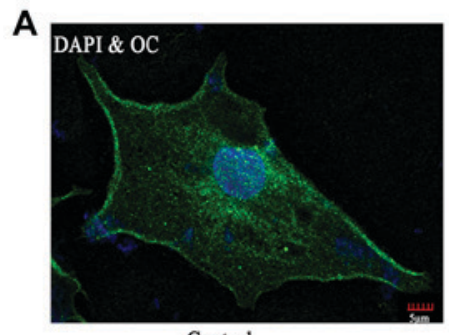

Control

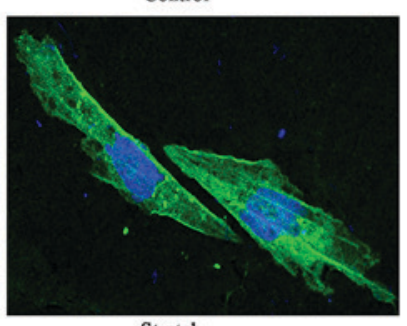

Stretch

D

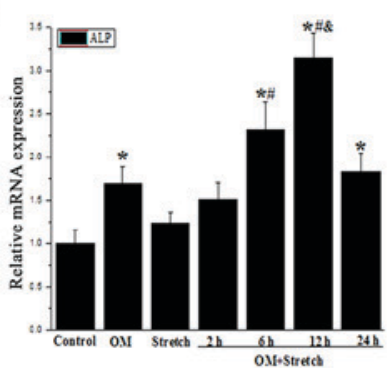

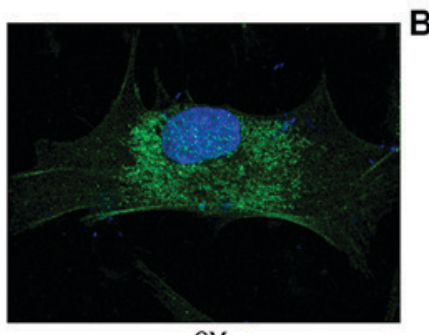

$\mathrm{OM}$

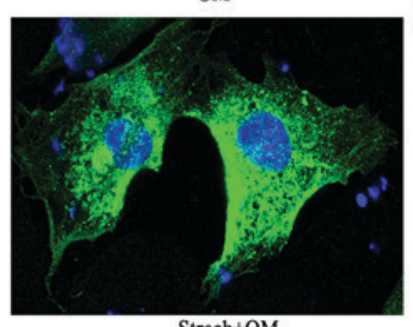

Strech $+0 \mathrm{M}$

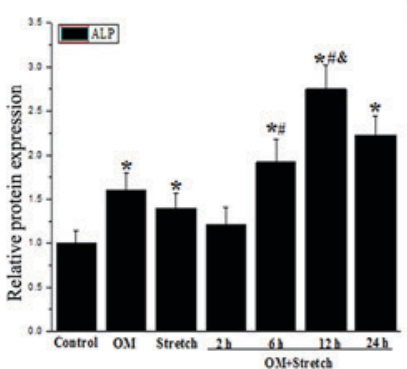

B
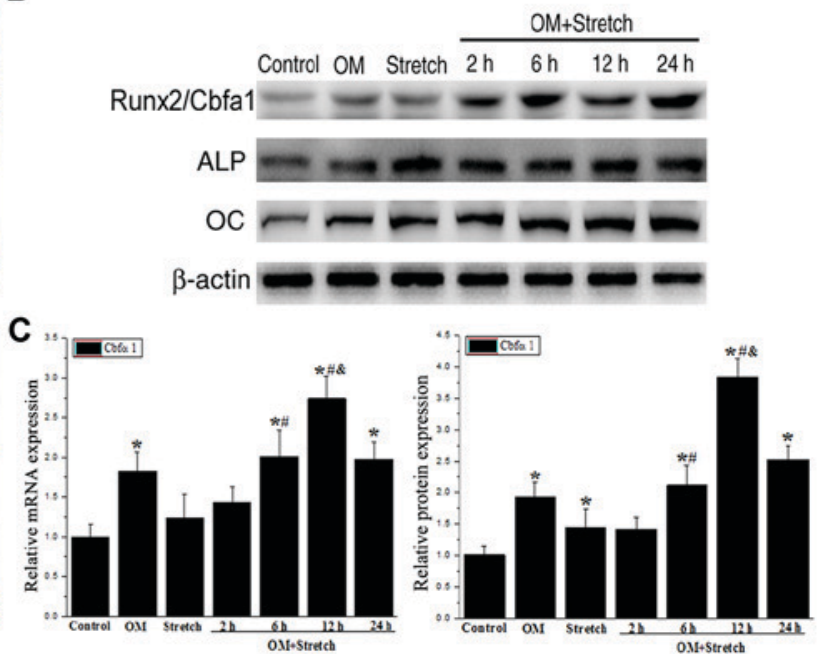

E
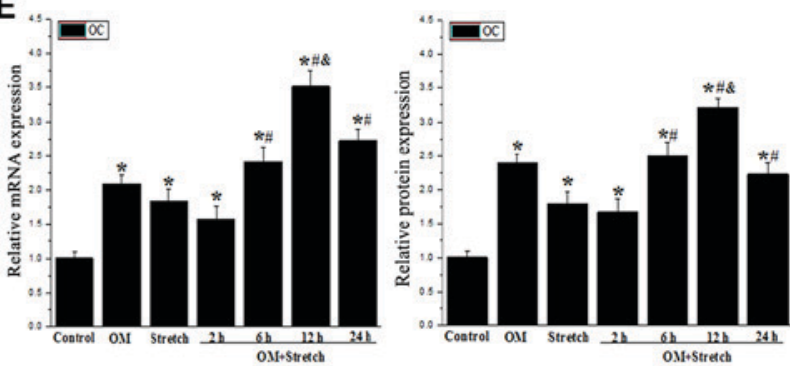

Figure 2. Quantitative polymerase chain reaction and western blot analysis for expression of Cbfa1, ALP and OC following different treatments. (A) The OC expression was increased at $12 \mathrm{~h}$ post-treatment in the $\mathrm{OM}$ and $\mathrm{OM}+$ stretch treatments (magnification, $\mathrm{x} 600$ ). (B) The mRNA and protein expression levels of (C) Runx2/Cbfa1 (D) ALP and (E) OC were increased significantly following treatment for 6, 12 and $24 \mathrm{~h}$ with the combined biochemical stimulation and stretching treatment but were decreased at $24 \mathrm{~h}(\mathrm{n}=5) .{ }^{*} \mathrm{P}<0.05$ vs. control, ${ }^{\#} \mathrm{P}<0.05$ vs. stretch and ${ }^{\&} \mathrm{P}<0.05$ vs. OM. OC, osteocalcin; ALP, alkaline phosphatase; OM, osteogenic induction medium; Runx2, Runt-related transcription factor 2; Cbfa1, core binding factor $\alpha 1$.

at $12 \mathrm{~h}$ post-treatment by $\mathrm{OM}$ and $\mathrm{OM}+$ stretch compared with the control (Fig. 2A). This indicated that hAECs can be induced to differentiate into osteogenic-like cells.

To investigate the whether that mechanical stretch induces hAECs to differentiate into osteoblasts, RT-qPCR and western blotting were performed to determine the expression levels of osteoblast markers, including Runx2/Cbfa1, alkaline phosphatase (ALP) and OC. The results suggested that the expression levels of Runx2/Cbfa1, ALP and OC demonstrated were significantly increased in the OM-induced group, the positive control group, and there was an increasing trend in the stretch group but with no significant differences compared with the control group (Fig. 2B-E).

Compared with the stretch or OM group alone, the mRNA and protein expression levels of Runx2/Cbfa1, ALP and OC increased following $6 \mathrm{~h}$ of treatment with OM combined with stretch $(\mathrm{OM}+$ stretch $)$ and peaked at $12 \mathrm{~h}$ but decreased at $24 \mathrm{~h}$ $(\mathrm{P}<0.05$; Fig. 2B-E). In brief, the above results suggested that the combination of $\mathrm{OM}$ and stretch had a synergistic effect on the osteogenic differentiation of hAECs.

Mechanical and biochemical stimulation modulates the osteogenic differentiation of $h A E C s$ via the Wnt/ $\beta$-catenin signalling pathway. The $\mathrm{Wnt} / \beta$-catenin signalling pathway has been known to affect the bone formation and homeostasis. A previous report indicated that the $\mathrm{Wnt} / \beta$-catenin signalling pathway enhanced osteogenic differentiation from human periodontal ligament fibroblasts (18). In the present study, the mRNA and protein expression levels of $\beta$-catenin and cyclin D1 were assessed following different treatments to confirm whether $\mathrm{Wnt} / \beta$-catenin signalling also has an important role in the osteogenic differentiation of hAECs. As shown in Fig. 3, the mRNA and protein expression levels of $\beta$-catenin, Runx2/Cbfa1 and cyclin D1 were significantly increased in the OM group $(\mathrm{P}<0.05)$. Furthermore, the combined application of stretching (the cells were stretched for $12 \mathrm{~h}$ ) with OM led to significant upregulation of $\beta$-catenin, Runx $2 / \mathrm{Cbfa} 1$ and cyclin D1 expression compared with the other treatments $(\mathrm{P}<0.05)$. This suggested that the Wnt $/ \beta$-catenin signalling pathway may mediate the osteogenic differentiation of AECs.

Based on these results, a Runx2-targeting shRNA was constructed and transfected into hAECs prior to $\mathrm{OM}$ or stretch stimulation to further investigate the mechanism of the Wnt/ $\beta$-catenin signalling pathway in osteoblastic differentiation of hAECs. As exhibited in Fig. 4A, the mRNA and protein levels of Runx2/Cbfa1 were decreased by $\sim 50$ and $70 \%$, respectively, following Runx2-shRNA transfection. The gene and protein levels of $\beta$-catenin and cyclin D1, as detected by RT-qPCR and western blotting, were significantly decreased following transfection of Runx2-shRNA in the basal and $\mathrm{OM} /$ stretch-stimulated groups $(\mathrm{P}<0.05$; Fig. 4B).

To examine whether Runx2-shRNA transfection could inhibit the differentiation process, confocal microscopy was used to assess the expression of OC in hAECs. The 

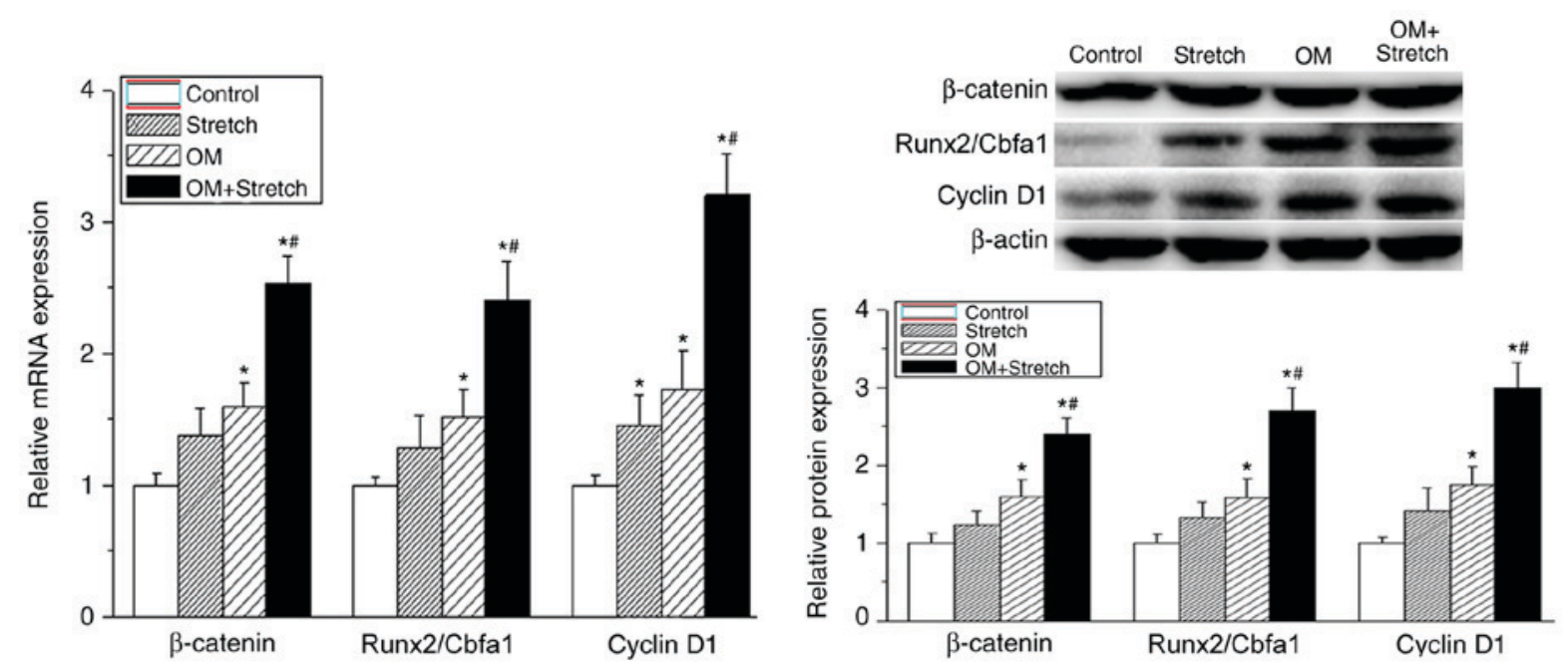

Figure 3. Quantitative polymerase chain reaction and western blot analysis for the expression of $\beta$-catenin, Runx2/Cbfa1 and cyclin D1 following stretch and OM. The mRNA and protein expression levels of $\beta$-catenin, Runx2/Cbfa1 and cyclin D1 were significantly upregulated following OM, stretch or OM + stretch and the levels in the $\mathrm{OM}+$ stretch group were increased compared the control group or the OM or stretch alone groups (time of cells stretched was $12 \mathrm{~h}$ ). ${ }^{*} \mathrm{P}<0.05$ vs. control, ${ }^{\sharp} \mathrm{P}<0.05$ vs. stretch. OM, osteogenic induction medium; Runx 2 , Runt-related transcription factor 2 ; Cbfa1, core binding factor $\alpha 1$.

immunocytochemistry results in Fig. 4C demonstrated that the OC expression was decreased in the transfected group compared with the control group, in the basal and stimulated groups, demonstrating that the osteogenic differentiation of AECs was blocked by cutting off the Wnt/ $\beta$-catenin signalling pathway. These results demonstrated that combined OM and stretch had an important role in the modulation of osteogenic differentiation of AECs via the Wnt/ $\beta$-catenin signalling pathway.

\section{Discussion}

The present study revealed certain significant results: i) Among all the treatment groups, the combined application of mechanical stretch and biochemical osteogenic stimulation of the hAECs has the most intense effects on gene or protein expression; and ii) the $\mathrm{Wnt} / \beta$-catenin signalling pathway had an important role in the osteogenic differentiation of the hAECs induced by stretch and/or biochemical stimulation. These results suggest that mechanical stretch, particularly when combined with biochemical stimulation, had an important role in the modulation of osteogenic differentiation of hAECs. Wnt/ $\beta$-catenin signalling leads to the differentiation of the hAECs into osteogenic lineage following mechanical stretch stimulation of osteogenic transcription factors.

In present study, the hAECs derived from full-term placenta seem to retain the capability to differentiate and to produce cell types from all three germ layers. Furthermore, hAECs are considered an ideal source of stem cells because of their ability to produce growth factors and catecholamines, and to differentiate into specific lineages and there are few concerns about their limitations $(5,6)$. These characteristics make the hAECs good candidates for use in the present study.

Previous studies have demonstrated that stem cell differentiation is affected by various factors, including the following: Static stretching increases cell proliferation and activates synergistic osteoblastic differentiation of mesenchymal cells; transforming growth factor- $\beta 1$ induced by microenvironments adjacent to distracted areas in vivo is thought to be involved in the regulation of bone synthesis and turnover (19); the renin-angiotensin system has been reported to mediate vascular endothelial growth factor synthesis in MSCs (20); biochemical stimulation has been reported to mediate the differentiation of hAECs into osteoblasts (12); cyclic stretching has been reported to promote osteogenesis-associated gene expression in osteoblast-like cells through a cofilin-associated mechanism mechanical stimuli (21); and mechanical loading is known to be an important factor in regulating bone growth and development (22). Thus, in the present study, it was hypothesized that hAECs differentiate into osteoblasts following stimulation by mechanical stretching.

The results of the present study indicate that treating cultured AECs with mechanical stretching altered cell morphology and modestly increased the gene and protein expression levels of Runx2/Cbfa1, ALP and OC in the hAECs. However, the combined application of stretch with OM led to further induction of Runx2/Cbfa1, ALP and OC gene and protein expression compared with either treatment alone, which demonstrated that combining stretch and biochemical osteogenic stimulation was synergistic for the osteogenic differentiation of the hAECs.

The expression of $\beta$-catenin in vitro and in vivo may contribute to the enhanced osteogenic differentiation of MSCs and bone formation in vivo $(19,20,23,24)$. Under physiological conditions, cytoplasmic $\beta$-catenin is maintained at low levels by a continuous process of phosphorylation-dependent ubiquitination and degradation in the absence of Wnt ligand. Upon Wnt stimulation, Wnt binds with a membrane receptor called frizzled, leading to the dimerization of membrane and cytoplasmic proteins. As a result, the kinases that phosphorylate and destabilize $\beta$-catenin are inhibited, and $\beta$-catenin then accumulates in the nucleus, where it associates with transcription factors such as T-cell-specific transcription factor/lymphoid enhancing factor to activate the expression of Wnt-responsive genes, including Runx2 and cyclin D (24-27). In the present study, the expression levels of $\beta$-catenin and cyclin D1 were 
A
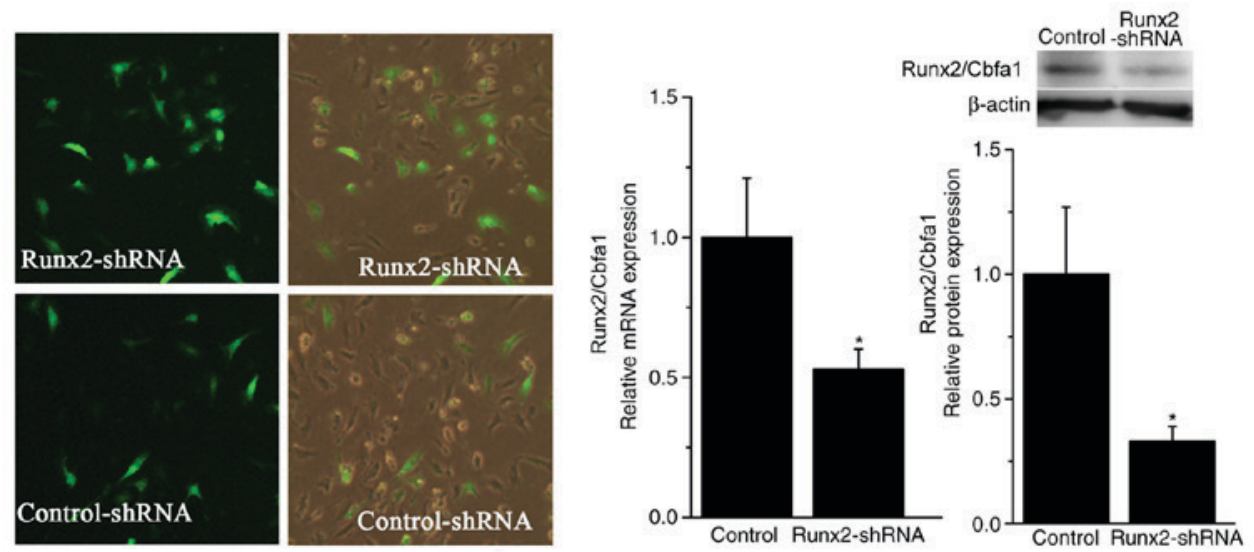
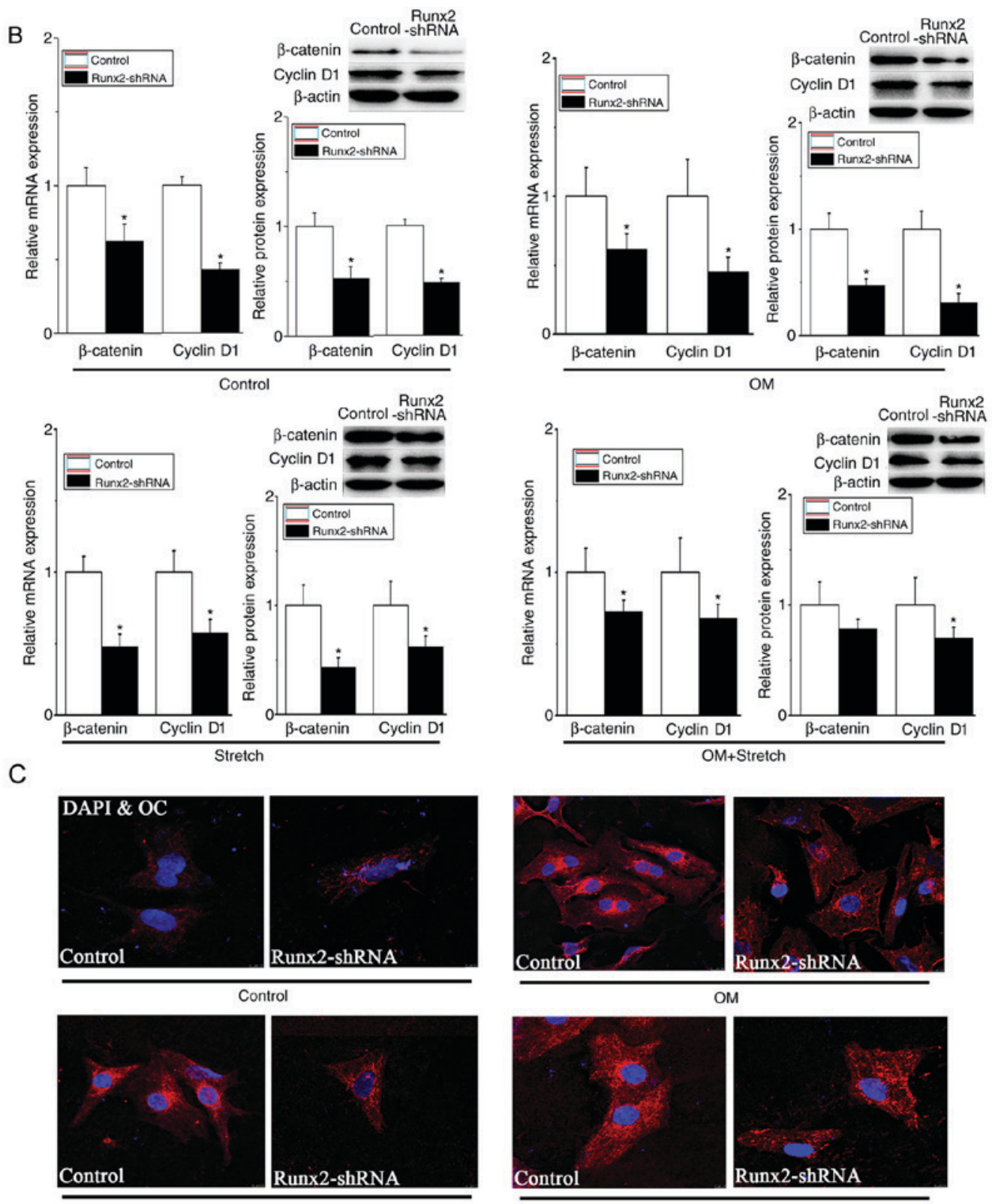

Stretch

OM+Stretch

Figure 4. Quantitative polymerase chain reaction and western blot analysis for the expression of $\beta$-catenin, Runx2/Cbfa1 and cyclin D1 following stretch and OM, and Runx2-shRNA transfection. (A) Runx2-shRNA was successfully transfected into hAECs and captured by fluorescence microscopy (x100). Green fluorescent protein is encoded by the shRNA vector and therefore GFP fluorescence was visualized as a surrogate for the infection efficiency and was demonstrated to be $47 \%$. (B) The mRNA and protein expression levels of $\beta$-catenin, Runx2/Cbfa1 and cyclin D1 decreased significantly following transfection of Runx2-shRNA in both the basal and stimulated groups; $(n=3)$. (C) The osteocalcin expression was decreased following transfection of Runx2-shRNA (x600). ${ }^{*} \mathrm{P}<0.05$ vs. control. OM, osteogenic induction medium; Runx2, Runt-related transcription factor 2 ; Cbfa1, core binding factor $\alpha 1$; shRNA, short hairpin RNA. 
upregulated following osteogenic differentiation of hAECs induced by mechanical stretching. Furthermore, combined stretching and biochemical stimulation induced the expression of $\beta$-catenin and cyclin D1.

Runx $2 / C b f a 1$, the runt domain-containing transcription factor, is a bone-specific product of the Cbfal gene that is essential for bone formation. It is one of the earliest and most specific markers during osteogenesis, and induces osteoblast-specific gene expression in vitro (28-30). The expression of Runx $2 / \mathrm{Cbfa} 1$ in response to different stimuli was examined, and the data demonstrated that the gene and protein expression was induced by the combination of mechanical and biochemical stimulation. However, the upregulation of Runx2/Cbfa1 was decreased by shRNA targeting Runx2/Cbfa1 into hAECs, as was the expression of $\beta$-catenin and cyclin D1. Notably, the OC protein level, as examined by confocal microscopy, was decreased following shRNA knockdown of Runx2/Cbfa1. Thus, the present observations demonstrated that mechanical stretching, and the combination of stretching and biochemical stimuli may induce the osteogenic differentiation of hAECs via activation of $\mathrm{Wnt} / \beta$-catenin signalling.

In conclusion, the results of the present study demonstrated that the combined use of mechanical stretching and biochemical stimulation was synergistic for the osteogenic differentiation of hAECs, and that this differentiation may be partially mediated via the Wnt/ $\beta$-catenin signalling pathway. Modulation of this may be a novel approach in bone regenerative medicine.

\section{Acknowledgements}

Not applicable.

\section{Funding}

The present study was supported by the Yongchuan Science and Technology Commission (grant no. YCSTC, 2015nc5006).

\section{Availability of data and materials}

The analysed data sets generated during the study are available from the corresponding author on reasonable request.

\section{Authors' contributions}

FL and HL designed experiments; KM, MZ and FY carried out experiments; JM analysed results. FL, HL and KM wrote the manuscript.

\section{Ethics approval and consent to participate}

All patients gave written informed consent. The present study was performed according to the Declaration of Helsinki and approved by the Medical Ethics Committee of the Yongchuan Hospital of Chongqing Medical University.

\section{Patient consent for publication}

Patients gave their consent for the present data to be published.

\section{Competing interests}

The authors declare that they have no competing interests.

\section{References}

1. Duncan RL and Turner CH: Mechanotransduction and the functional response of bone to mechanical strain. Calcif Tissue Int 57: 344-358, 1995.

2. Qi MC, Zou SJ, Han LC, Zhou HX and Hu J: Expression of bone-related genes in bone marrow MSCs after cyclic mechanical strain: Implications for distraction osteogenesis. Int J Oral Sci 1: 143-150, 2009

3. Maul TM, Chew DW, Nieponice A and Vorp DA: Mechanical stimuli differentially control stem cell behavior: Morphology, proliferation, and differentiation. Biomech Model Mechanobiol 10: 939-953, 2011.

4. Ilancheran S, Moodley Y and Manuelpillai U: Human fetal membranes: A source of stem cells for tissue regeneration and repair? Placenta 30: 2-10, 2009.

5. Miki T, Mitamura K, Ross MA, Stolz DB and Strom SC: Identification of stem cell marker-positive cells by immunofluorescence in term human amnion. J Reprod Immunol 75: 91-96, 2007.

6. Toda A, Okabe M, Yoshida T and Nikaido T: The potential of amniotic membrane/amnion-derived cells for regeneration of various tissues. J Pharmacol Sci 105: 215-228, 2007.

7. Hodge A, Lourensz D, Vaghjiani V, Nguyen H, Tchongue J, Wang B, Murthi P, Sievert W and Manuelpillai U: Soluble factors derived from human amniotic epithelial cells suppress collagen production in human hepatic stellate cells. Cytotherapy 16: 1132-1144, 2014.

8. Niknejad H, Peirovi H, Ahmadiani A, Ghanavi J and Jorjani M: Differentiation factors that influence neuronal markers expression in vitro from human amniotic epithelial cells. Eur Cells Mater 19: 22-29, 2010.

9. Kakishita K, Elwan MA, Nakao N, Itakura T and Sakuragawa N: Human amniotic epithelial cells produce dopamine and survive after implantation into the striatum of a rat model of Parkinson's disease: A potential source of donor for transplantation therapy. Exp Neurol 165: 27-34, 2000.

10. Wlodarski K, Moskalewski S, Skarzyńska S, Póltorak A and Ostrowski K: Irradiation and the bone induction properties of epithelial cells. Bull Acad Pol Sci Biol 19: 821-825, 1971.

11. Wang Q, Wu W, Han X, Zheng A, Lei S, Wu J, Chen H, He C, Luo F and Liu X: Osteogenic differentiation of amniotic epithelial cells: Synergism of pulsed electromagnetic field and biochemical stimuli. BMC Musculoskelet Disord 15: 271, 2014.

12. Parolini O, Alviano F, Bagnara GP, Bilic G, Bühring HJ, Evangelista M, Hennerbichler S, Liu B, Magatti M, Mao N, et al: Concise review: Isolation and characterization of cells from human term placenta: Outcome of the first international workshop on placenta derived stem cells. Stem Cells 26: 300-311, 2008.

13. Miki T, Marongiu F, Dorko K, Ellis EC and Strom SC: Isolation of amniotic epithelial stem cells. Curr Protoc Stem Cell Biol Chapter 1: Unit 1E.3, 2010.

14. Hata M, Naruse K, Ozawa S, Kobayashi Y, Nakamura N, Kojima N, Omi M, Katanosaka Y, Nishikawa T, Naruse K, et al: Mechanical stretch increases the proliferation while inhibiting the osteogenic differentiation in dental pulp stem cells. Tissue Eng Part A 19: 625-633, 2013.

15. Yang YQ, Li XT, Rabie AB, Fu MK and Zhang D: Human periodontal ligament cells express osteoblastic phenotypes under intermittent force loading in vitro. Front Biosci 1: 776-781, 2006.

16. Koike M, Shimokawa H, Kanno Z, Ohya K and Soma K: Effects of mechanical strain on proliferation and differentiation of bone marrow stromal cell line ST2. J Bone Miner Metab 23: 219-225, 2005.

17. Livak KJ and Schmittgen TD: Analysis of relative gene expression data using real-time quantitative PCR and the 2(-Delta Delta C(T)) method. Methods 25: 402-408, 2001.

18. Heo JS, Lee SY and Lee JC: Wnt//-catenin signaling enhances osteoblastogenic differentiation from human periodontal ligament fibroblasts. Mol Cells 30: 449-454, 2010.

19. Kim IS, Song YM and Hwang SJ: Osteogenic responses of human mesenchymal stromal cells to static stretch. J Dent Res 89: 1129-1134, 2010. 
20. Liu C, Zhang JW, Hu L, Song YC, Zhou L, Fan Y, Zhu HY,

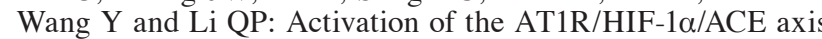
mediates angiotensin II-induced VEGF synthesis in mesenchymal stem cells. Biomed Res Int 2014: 627380, 2014.

21. Gao J, Fu S, Zeng Z, Li F, Niu Q, Jing D and Feng X: Cyclic stretch promotes osteogenesis-related gene expression in osteoblast-like cells through a cofilin-associated mechanism. Mol Med Rep 14: 218-224, 2016

22. Wu Y, Zhang P, Dai Q, Yang X, Fu R, Jiang L and Fang B: Effect of mechanical stretch on the proliferation and differentiation of BMSCs from ovariectomized rats. Mol Cell Biochem 382: 273-282, 2013.

23. Dao DY, Jonason JH, Zhang Y, Hsu W, Chen D, Hilton MJ and O'Keefe RJ: Cartilage-specific $\beta$-CATENIN signaling regulates chondrocyte maturation, generation of ossification centers, and perichondrial bone formation during skeletal development. J Bone Miner Res 27: 1680-1694, 2012.

24. Jian H, Shen X, Liu I, Semenov M, He X and Wang XF: Smad3-dependent nuclear translocation of beta-catenin is required for TGFbetal-induced proliferation of bone marrow-derived adult human mesenchymal stem cells. Genes Dev 20: 666-674, 2006.

25. Kestler HA and Kühl M: From individual Wnt pathways towards a Wnt signaling network. Philos Trans R Soc Lond B Biol Sci 363: 1333-1347, 2008.
26. Taurin S, Sandbo N, Qin Y, Browning D and Dulin NO: Phosphorylation of beta-catenin by cyclic AMP-dependent protein kinase. J Biol Chem 281: 9971-996, 2006.

27. Takemaru KI and Moon RT: The transcriptional eoaetivator CBP interaets with beta-catenin to activate gene expression. J Cell Biol 149: 249-254, 2000.

28. Komori T, Yagi H, Nomura S, Yamaguchi A, Sasaki K, Deguchi K, Shimizu Y, Bronson RT, Gao YH, Inada M, et al: Targeted disruption of Cbfa1 results in a complete lack of bone formation owing to maturational arrest of osteoblasts. Cell 89: 755-764, 1997.

29. Xiao G, Jiang D, Ge C, Zhao Z, Lai Y, Boules H, Phimphilai M, Yang X, Karsenty G and Franceschi RT: Cooperative interactions between activating transcription factor 4 and Runx2/Cbfa1 stimulate osteoblast-specific osteocalcin gene expression. J Biol Chem 280: 30689-30696, 2005.

30. Mundlos S, Otto F, Mundlos C, Mulliken JB, Aylsworth AS, Albright S, Lindhout D, Cole WG, Henn W, Knoll JH, et al: Mutations involving the transcription factor CBFA1 cause cleidocranial dysplasia. Cell 89: 773-779, 1997. 